\title{
Topology optimization of trusses using bars exchange method
}

\author{
D. BOJCZUK* and A. RĘBOSZ-KURDEK \\ Faculty of Management and Computer Modelling, Kielce University of Technology \\ 7 Tysiąclecia Państwa Polskiego Ave., 25-314 Kielce, Poland
}

\begin{abstract}
The algorithm of optimization of trusses is presented in the paper, where for topology optimization the bars exchange method is used. In the first case, the problem aimed at cost minimization with a constraint set on global stiffness is formulated. In the second case, the problem of minimizing the cost function subjected to stress and cross-sectional area constraints is discussed and here the multiple-load case is taken into consideration. The conditions for introduction of topology modification and its acceptance are specified. The paper is illustrated with three examples.
\end{abstract}

Key words: trusses, algorithm of optimization, optimal topologies, bars exchange.

\section{Introduction}

The problem of optimal design of trusses is considered in this paper. Here the two-stage (topology and geometry) optimization procedure was presented, however attention was mainly focused on topology optimization.

General classification of topology optimization methods on reduction and expansion processes was proposed in [1].

Topological optimization is usually based on reduction process, which is characterized by elimination of members and joints from an initial highly connected ground structure (structural universe). The ground structure approach is actually regarded as the standard method for truss topology design. It was established in [2] and next developed in [3,4], etc. The simultaneous optimization of truss geometry and topology, based on ground structure approach is analyzed in [5].

The expansion process is characterized by the addition of members and joints to an initial structural topology, cf. [6$8]$, etc. In the approach described in [6], it is assumed that, similarly to biological growth models, the structure evolves with the characteristic size parameter and a "bifurcation" of topology occurs with the generation of new nodes, in order to minimize the cost function.

The topology optimization method presented in this paper can be classified as a process without the change of structure complexity degree because it is assumed that the number of members and joints is constant. Here, bars exchange method is proposed. At first, using topological derivative, additional virtual bars are selected. Next, including existing and virtual bars, statically admissible fields of bar forces are created in order to minimize the objective function and delete redundant bars. This method is the extension of the approach formulated in [9] and [10]. Let us notice, that topological derivative for trusses (treated as graphs) is also applied in [11].

The problem formulation, optimality conditions and topology modification conditions will be derived in Sec. 2. In Sec. 3 the algorithm of optimal truss design will be discussed and in Sec. 4 the illustrative examples confirming applicability of proposed approach will be presented and non-uniqueness of optimal topologies is shown, cf. [12].

\section{Problem formulation, optimality conditions and topology modification condition}

2.1. Minimum cost problem with stiffness constraints. The problem of optimal design for trusses requiring minimum of material cost with constraint set on global stiffness, can be written as

$$
\min C=\sum_{i=1}^{n} c_{i} A_{i} l_{i} \text { subject to } U=\frac{1}{2} \sum_{i=1}^{n} \frac{N_{i}^{2} l_{i}}{E_{i} A_{i}} \leq U_{0},
$$

where $C$ is the total cost of the structure, $A_{i}, l_{i}, c_{i}, E_{i}$ are the cross-sectional area, length, specific material cost and Young's modulus of the $i$-th bar, $U$ denotes strain energy and $U_{0}$ is the allowable strain energy.

Using the Lagrangian

$$
C^{*}=C+\lambda\left(U-U_{0}\right)
$$

we obtain the optimality conditions, with respect to crosssectional areas and Lagrange multiplier $\lambda(\lambda \geq 0)$, in the form

$$
\begin{gathered}
\frac{\partial C^{*}}{\partial A_{i}}=c_{i} l_{i}-\frac{1}{2} \lambda \frac{N_{i}^{2} l_{i}}{E_{i} A_{i}^{2}}=\left(c_{i}-\frac{1}{2} \lambda \varepsilon_{i}^{2} E_{i}\right) l_{i}=0, \\
\lambda\left(\frac{1}{2} \sum_{i=1}^{n} \frac{N_{i}^{2} l_{i}}{E_{i} A_{i}}-U_{0}\right)=0, \quad \lambda \geq 0,
\end{gathered}
$$

where the following sensitivity formula, derived in view of the equilibrium equations, was used, cf. [13]

$$
\frac{\partial U}{\partial A_{i}}=-\frac{N_{i}^{2} l_{i}}{2 E_{i} A_{i}^{2}}
$$

\footnotetext{
*e-mail: mecdb@tu.kielce.pl
} 
Calculating from (3), cross-sectional areas and Lagrange multiplier in function of bar forces, we have

$$
\begin{gathered}
A_{i}=\frac{1}{2 U_{0}} \frac{\left|N_{i}\right|}{\sqrt{c_{i} E_{i}}} \sum_{j=1}^{n}\left|N_{j}\right| l_{j} \sqrt{\frac{c_{j}}{E_{j}}} \\
\lambda=\left(\frac{1}{U_{0}} \sum_{i=1}^{n}\left|N_{i}\right| l_{i} \sqrt{\frac{c_{i}}{2 E_{i}}}\right)^{2} .
\end{gathered}
$$

Now, the cost function, both for the statically determinate and non-determinate trusses, can be expressed as

$$
C=\frac{1}{2 U_{0}}\left(\sum_{i=1}^{n}\left|N_{i}\right| l_{i} \sqrt{\frac{c_{i}}{E_{i}}}\right)^{2} .
$$

When all bars are made of the same material, so that

$$
E=E_{1}=E_{2}=\ldots=E_{n}, \quad c=c_{1}=c_{2}=\ldots=c_{n},
$$

the cost function has the form

$$
C=\frac{c}{2 E U_{0}}\left(\sum_{i=1}^{n}\left|N_{i}\right| l_{i}\right)^{2}
$$

Using topological derivative approach, the condition of topology modification by introduction of a new $(n+1)$-th bar of Young's modulus $E_{n+1}$ and specific cost $c_{n+1}$ to the truss with optimal cross-sectional areas determined from $(5)_{1}$, can be expressed as follows

$$
\left.\frac{\partial C^{*}}{\partial A_{n+1}}\right|_{A_{n+1}=0}<0, \quad \text { or } \quad\left|\varepsilon_{n+1}\right|>\varepsilon_{b}^{(n+1)},
$$

where

$$
\varepsilon_{b}^{(n+1)}=\sqrt{\frac{2 c_{n+1}}{\lambda E_{n+1}}} \text { and } \varepsilon_{n+1}
$$

denotes virtual strain value along the line connecting the respective nodes. Fulfillment of the condition (9) means, that after introduction of a new virtual bar, decrease of the cost function will appear. When all bars are made of the same material, the condition (9) takes the form

$$
\left|\varepsilon_{n+1}\right|>\varepsilon_{b}, \quad \text { where } \quad \varepsilon_{b}=\left|\varepsilon_{1}\right|=\left|\varepsilon_{2}\right|=\ldots=\left|\varepsilon_{n}\right| .
$$

2.2. Minimum cost problem with stress and crosssectional area constraints. The problem of the optimal design for trusses requiring minimum of the material cost with stress and cross-sectional area constraints, is formulated in the form

$$
\begin{aligned}
& \min C=\sum_{i=1}^{n} c_{i} A_{i} l_{i} \quad \text { subject to } \\
& \left|\sigma_{i}^{(j)}\right| \leq \sigma_{a i}^{(j)}, \quad A_{i} \geq A_{i \min },
\end{aligned}
$$

in which the notation is the same as in Subsec. 2.1 and $j$ $\left(j=1, \ldots, j_{0}\right)$ denotes number of loading case. Furthermore, $\sigma_{i}^{(j)}=N_{i}^{(j)} / A_{i}$ are the current stresses, while

$$
\sigma_{a i}^{(j)}= \begin{cases}R_{e i} & \text { for } \sigma_{i}^{(j)} \geq 0 \\ \frac{\pi^{2} E_{i}}{s_{i}^{2}} & \text { for } \sigma_{i}^{(j)}<0, s_{i} \geq s_{i}^{c r} \\ R_{e i}-\frac{1}{2} R_{e i}\left(\frac{s_{i}}{s_{i}^{c r}}\right)^{2} & \text { for } \sigma_{i}^{(j)}<0, s_{i}<s_{i}^{c r}\end{cases}
$$

denote the admissible stresses in the $i$-th bar for the $j$-th loading case and $R_{e i}$ is the conventional yield limit. The last expression of (12) corresponds to the Johnson-Ostenfeld formula for the critical buckling stress in the elasto-plastic range, which also follows from Shanley's tangent modulus theory presented in [14]. Moreover,

$$
s_{i}=l_{i} \sqrt{\frac{A_{i}}{I_{i}}}, \quad s_{i}^{c r}=\pi \sqrt{\frac{2 E_{i}}{R_{e i}}}
$$

denote the slenderness and critical slenderness values, and $I_{i}$ is the minimal moment of inertia of the bar cross-section.

We assume the relation between the moment of inertia and the cross-sectional area in the form

$$
I=\xi A^{m}, \quad m=1,2,3,
$$

where $\xi$ is the constant parameter. Let us notice, that the case $m=2$ corresponds to the proportional change of the crosssectional dimensions. Accounting for (13) and (14) in (12) the admissible stresses take the form

$$
\sigma_{a i}^{(j)}= \begin{cases}R_{e i} & \text { for } \sigma_{i}^{(j)} \geq 0 \\ \frac{\pi^{2} E_{i} \xi_{i} A^{m-1}}{l_{i}^{2}} & \text { for } \sigma_{i}^{(j)}<0, s_{i} \geq s_{i}^{c r} \\ R_{e i}-\frac{R_{e i}^{2}}{4 E_{i} \pi^{2}} \frac{l_{i}^{2}}{\xi_{i} A_{i}^{m-1}} & \text { for } \sigma_{i}^{(j)}<0, s_{i}<s_{i}^{c r}\end{cases}
$$

and the derivatives of admissible stresses are

$$
\frac{\partial \sigma_{a i}^{(j)}}{\partial A_{i}}= \begin{cases}0 & \text { for } \sigma_{i}^{(j)} \geq 0 \\ \frac{(m-1) \pi^{2} E_{i} \xi_{i} A^{m-2}}{l_{i}^{2}} & \text { for } \sigma_{i}^{(j)}<0, s_{i} \geq s_{i}^{c r} \\ \frac{(m-1) R_{e i}^{2} l_{i}^{2}}{4 E_{i} \pi^{2} \xi_{i} A_{i}^{m}} & \text { for } \sigma_{i}^{(j)}<0, s_{i}<s_{i}^{c r}\end{cases}
$$

Introducing nonnegative Lagrange multipliers $\mu_{i} \geq 0$, $\eta_{i} \geq 0$, the augmented objective function can be expressed as

$$
\begin{gathered}
C^{*}\left(A_{i}, \mu_{i}, \eta_{i}\right)=C+\sum_{i=1}^{n} \mu_{i}\left(\left|\sigma_{i}^{(j)}\right|-\sigma_{a i}^{(j)}\right) \\
+\sum_{i=1}^{n} \eta_{i}\left(A_{\min }-A_{i}\right) .
\end{gathered}
$$

Now, the optimality conditions, with respect to crosssectional areas and Lagrange multipliers, take the form

$$
\begin{gathered}
\frac{\partial C^{*}}{\partial A_{i}}=c_{i} l_{i}+\mu_{i}\left(\frac{\partial\left|\sigma_{i}^{(j)}\right|}{\partial A_{i}}-\frac{\partial \sigma_{a i}^{(j)}}{\partial A_{i}}\right)-\eta_{i}=0, \quad i=1, \ldots, n, \\
\mu_{i}\left(\left|\sigma_{i}^{(j)}\right|-\sigma_{a i}^{(j)}\right)=0, \quad i=1, \ldots, n, \\
\eta_{i}\left(A_{\min }-A_{i}\right)=0, \quad i=1, \ldots, n .
\end{gathered}
$$

In order to solve (18) with respect to $A_{i}, \mu_{i}$ and $\eta_{i}$ we can divide bars into four groups, namely: 
- group 1 corresponding to tensile bars of cross-sectional areas greater than $A_{\min }$;

- group 2 corresponding to compressive bars of slenderness not less than the critical slenderness $s_{i}^{c r}$ and of crosssectional areas greater than $A_{\text {min }}$;

- group 3 corresponding to compressive bars of slenderness less than the critical slenderness $s_{i}^{c r}$ and of cross-sectional areas greater than $A_{\min }$;

- group 4 of bars of cross-sectional areas $A=A_{\text {min }}$.

Taking $m=2$ and limiting considerations to the class of statically determinate trusses, we get:

- for group 1

$$
A_{i}=\frac{N_{i}^{w}}{R_{e i}}, \quad \mu_{i}=\frac{c_{i}}{R_{e i}^{2}} N_{i}^{w} l_{i}, \quad \eta_{i}=0 ;
$$

- for group 2

$$
A_{i}=\frac{\sqrt{\left|N_{i}^{w}\right|} l_{i}}{\pi \sqrt{E_{i} \xi_{i}}}, \quad \mu_{i}=\frac{c_{i} l_{i}^{3}}{2 \pi^{2} E_{i} \xi_{i}}, \quad \eta_{i}=0
$$

- for group 3

$$
\begin{gathered}
A_{i}=\frac{\left|N_{i}^{w}\right|}{R_{e i}}+\frac{R_{e i} l_{i}^{2}}{4 E_{i} \pi^{2} \zeta_{i}}, \\
\mu_{i}=\frac{c_{i} l_{i}}{R_{e i}}\left(\left|N_{i}^{w}\right|+\frac{R_{e i}^{2} l_{i}^{2}}{4 E_{i} \pi^{2} \xi_{i}}\right), \quad \eta_{i}=0 ;
\end{gathered}
$$

- for group 4

$$
A_{i}=A_{\min }, \quad \mu_{i}=0, \quad \eta_{i}=c_{i} l_{i}
$$

where $N_{i}^{w}$ is the normal force in the $i$-th bar sizing its crosssectional area, corresponding to the worst loading case for this bar.

So, using (19)-(22), the cross-sectional areas $A_{i}$, in terms of bar forces, can be expressed as follows

$$
A_{i}=\left\{\begin{array}{l}
\frac{\left|N_{i}^{w}\right|}{R_{e i}}+\frac{R_{e i} l_{i}^{2}}{4 E_{i} \pi^{2} \xi_{i}}, \quad N_{i}^{w} \leq-\frac{l_{i}^{2} R_{e i}^{2}}{4 E_{i} \pi^{2} \xi_{i}} \\
\frac{\sqrt{\left|N_{i}^{w}\right|} l_{i}}{\pi \sqrt{E_{i} \xi_{i}}}, \quad-\frac{l_{i}^{2} R_{e i}^{2}}{4 E_{i} \pi^{2} \xi_{i}} \leq N_{i}^{w} \leq-\frac{\pi^{2} \xi_{i} E_{i} A_{i \min }^{2}}{l_{i}^{2}} \\
A_{i \min }, \quad-\frac{\pi^{2} \xi_{i} E_{i} A_{i \min }^{2}}{l_{i}^{2}} \leq N_{i}^{w} \leq R_{e i} A_{i \min } \\
\frac{1}{R_{e i}} N_{i}^{w}, \quad N_{i}^{w} \geq R_{e i} A_{i \min } .
\end{array}\right.
$$

Substituting (19)-(22) into the objective function (11), it can be expressed in the following form

$$
\begin{gathered}
C=\sum_{(1)} \frac{c_{i}}{R_{e i}} N_{i}^{w} l_{i}+\sum_{(1)} \frac{c_{i}}{\pi \sqrt{\xi_{i} E_{i}}} \sqrt{\left|N_{i}^{w}\right| l_{i}^{2}} \\
+\sum_{(2)} \frac{c_{i}}{R_{e i}}\left[\left|N_{i}^{w}\right| l_{i}+\frac{R_{e i}^{2} l_{i}^{3}}{4 E_{i} \pi^{2} \xi_{i}}\right]+\sum_{(4)} c_{i} A_{i \min } l_{i},
\end{gathered}
$$

where $\sum_{(i)}$ denotes the summation with respect to all bars belonging to group $i$.

The condition of topology modification by introduction of $(n+1)$-th bar in tension takes the form (9) or (10), where here
$\varepsilon_{b}^{(n+1)}=R_{e(n+1)} / E_{n+1}$. In the case of bar in compression the condition can be presented as

$$
\left|\varepsilon_{n+1}\right|>\varepsilon_{0_{n+1}}, \text { where } \varepsilon_{0_{n+1}}=\frac{\pi^{2} \xi_{n+1} A_{(n+1) \min }}{l_{n+1}^{2}}
$$

denotes strain appearing in the virtual bar in compression of the minimal cross-sectional area.

\section{The algorithm of optimal truss design}

The algorithm presented here is valid for the problems considered in Subsec. 2.1, and also in Subsec. 2.2, but it is limited to one loading case $\left(j_{0}=1\right)$. However, it can be easily generalized to many loading cases. The following steps can be distinguished in the solution algorithm.

1. Choice of initial truss of required complexity degree.

2. Truss analysis. Determination of bar forces can be executed using for example FEM. The analysis problem for a truss is specified by the equilibrium equations

$$
\mathbf{K u}=\mathbf{P},
$$

where $\mathbf{K}$ is the global stiffness matrix, $\mathbf{u}$ is the nodal displacement vector, and $\mathbf{P}$ is the load vector.

3. Redistribution of cross-sectional areas. The optimal cross-sectional areas $A_{i}$ for the problem (1) or (11) are calculated respectively from $(5)_{1}$ or $(23)$.

4. Choice of virtual bars. The virtual connections, where we can introduce new bars are determined using modification conditions (9), (10) or (25).

5. Topology optimization. When a new bar is introduced, in order to avoid increase of bars number, another bar should be deleted. It is executed through the procedure of bar exchange. Instead of each $J$-th virtual bar self-equilibrated force system $\alpha \widehat{N}_{J}$ is introduced, where $\widehat{N}_{J}$ is the unit force and $\alpha$ is the load factor. The unit force system induces in truss bars the normal forces $\alpha \widehat{N}_{i}$, which are next calculated.

The procedure of topology optimization is conducted by the iterative scheme consisting of the following steps:

5.1. Calculation of the total bar forces. The total bar forces are calculated from the following formula

$$
N_{i}^{c}=N_{i}+\alpha \widehat{N}_{i} \text {. }
$$

Using conditions $N_{i}^{c}=0$ we get values of parameter $\alpha$ corresponding to the disappearance of normal forces in consecutive bars.

5.2. Determination of the objective function variation. For each virtual bar and each value of parameter $\alpha$ the objective function variation is determined

$$
\Delta C=C^{(n)}-C^{(i)}<0,
$$

where $C^{(n)}$ denotes the value of $C$ corresponding to the new topology and $C^{(i)}$ is the value of $C$ at the previous step before topology variation. Under the condition $\Delta C<0$, the change (changes) corresponding to the biggest decrease of the $\Delta C$ is chosen.

5.3. Determination of bar forces after modification. For each bar exchange, that generate the biggest decrease of the objective function value, bar forces are determined analogously as in step 5.1 . 
5.4. Termination of the topology optimization procedure. If any bars exchange is not possible, i.e. $\Delta C \geq 0$ the next step is conducted, otherwise we go to step 5.1.

6. Configuration optimization. For given truss topology the optimization of truss configuration is performed.

7. Termination of the algorithm. If any modification of topology cannot be introduced the optimization procedure is terminated, otherwise we go to step 2.

\section{Examples}

4.1. Example 1. Optimization of topology and configuration of truss with respect to cost minimization under stiffness constraint. Consider the cost optimization problem of the form described by (1) for the truss presented in Fig. 1a. Three lower nodes of the truss are loaded by vertical forces $P, 2 P, P$, where $P=10^{3} \mathrm{~N}$. The truss consists of 17 bars and it is made of linearly elastic material of Young's modulus value $E=2.1 \cdot 10^{11} \mathrm{~N} / \mathrm{m}^{2}$. The length of all horizontal and vertical bars is $l=1 \mathrm{~m}$, while the length of inclined bars equals $\sqrt{2} l$. It is also assumed that specific material costs $c_{i}$ for all bars of the truss are the same.

a)

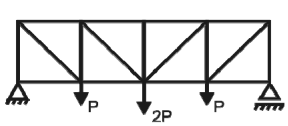

b)
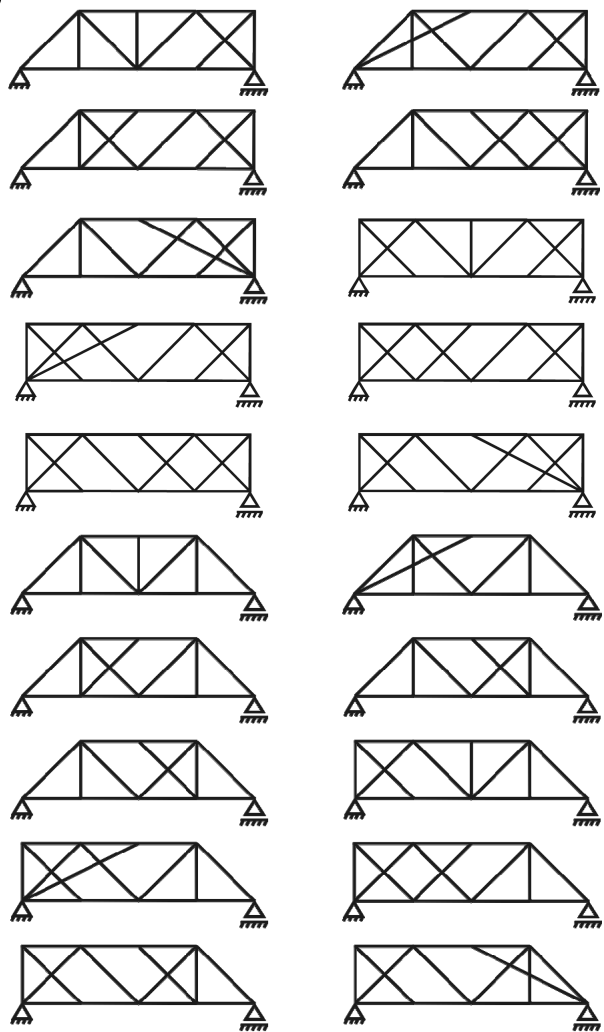

c)

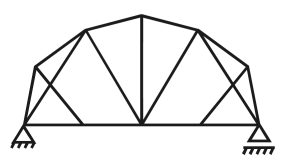

Fig. 1. Optimization of the truss: a) the initial design, b) selected optimal topologies, c) the optimal design
The topology optimization by bars exchanges gives 80 equivalent designs. For each design the objective function reaches the same value, so the solution is non-unique. The removal of zero bars system from optimal topologies, will decrease their number to 20 (Fig. 1b). After topology modification, the cost reduction is about $12 \%$. Finally, the optimal design (Fig. 1c) is obtained by configuration optimization of the optimal topologies. Now, the cost is about 33\% smaller than that of the initial design corresponding to the truss presented in Fig. 1a, with the optimal cross-sectional areas.

4.2. Example 2. Optimization of topology and configuration of truss with respect to cost minimization under stress and buckling constraints for one loading case. Consider the cost optimization problem of the form described by (11) for the 21-bars truss presented in Fig. 2a. Here, one loading case is analysed $\left(j_{0}=1\right)$, namely five upper nodes of the truss are loaded by vertical forces $P$ and five lower nodes by vertical forces $5 P$, where $P=10^{3} \mathrm{~N}$. The Young's modulus value is the same as in the previous example. The yield limit is $R_{e}=2.5 \cdot 10^{8} \mathrm{~N} / \mathrm{m}^{2}$. The minimal cross-sectional area is $A_{\text {min }}=0.2 \cdot 10^{-4} \mathrm{~m}^{2}$.

a)

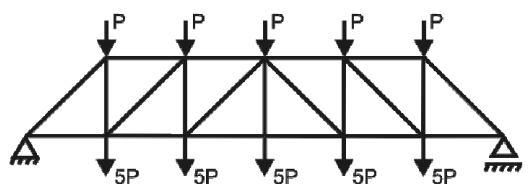

b)

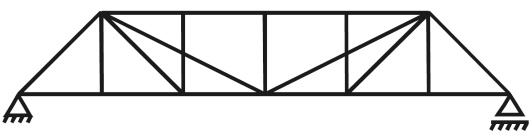

c)

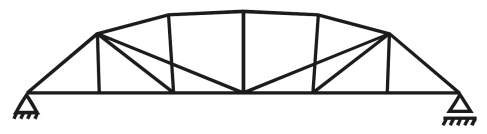

Fig. 2. Optimization of the truss: a) The initial design, b) The optimal topology, c) The optimal design

We assume that all cross-sections have circular shape and in this case, the coefficient of the relation between the moment of inertia and the cross-sectional area is $\xi=1 /(4 \pi)$. We also assume that specific material costs $c_{i}$ for all bars of the truss are the same.

The topology optimization by bars exchanges leads to the structure presented in Fig. 2b. Further cost reduction can be achieved by configuration optimization. The final optimal design is shown in Fig. 2c. Now, the cost is $30 \%$ smaller than that of the initial design (Fig. 2a), while topology optimization gives a cost decrease about $23 \%$.

4.3. Example 3. Optimization of topology and configuration of truss with respect to cost minimization under stress and buckling constraints for two loading cases. Let us consider now the cost optimization problem of the form described by (11), for the 15-bars truss (Fig. 3a) and two 
loading cases $\left(j_{0}=2\right)$. Details concerning applied loads are given in Table 1. The material parameters and the minimal cross-sectional area values are the same as in the previous example.

a)

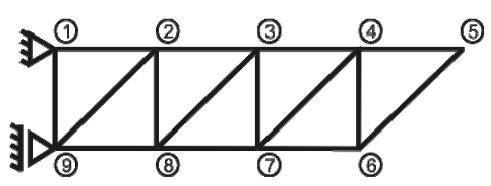

b)

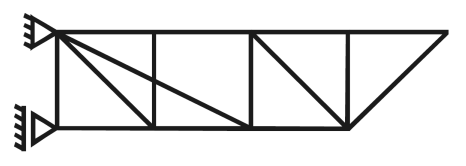

c)

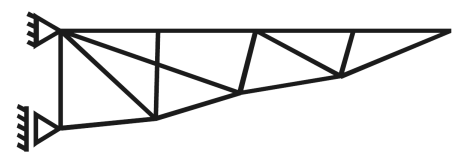

d)

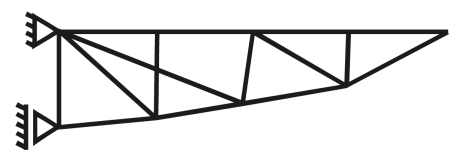

e)

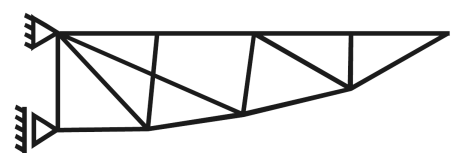

Fig. 3. Optimization of the truss: a) The initial design, b) The optimal topology, c) The optimal design for the first loading case, d) The optimal design for the second loading case, e) The optimal design for both loading cases

Table 1

Truss loading conditions, forces are given in $[\mathrm{N}]$

\begin{tabular}{cccc}
\hline \hline Load case & Node number & $P_{x}$ & $P_{y}$ \\
\hline 1 & 2 & 0 & -40000 \\
& 3 & 0 & -30000 \\
4 & 0 & -20000 \\
& 5 & 0 & -10000 \\
\hline 2 & 5 & 0 & -50000 \\
\hline
\end{tabular}

At first, the optimization procedure is carried out for each load case applied independently. Next, the multi-load case is taken into account and procedure is performed simultaneously for both load cases.

The topology optimization conducted by bars exchange method leads to the structure shown in Fig. 3b. The structure is the same for each considered loading cases. The configuration optimization provides the final optimal designs shown in Fig. 3c, $d$ and e. The percentage decrease of the cost value after topology and configuration optimization with relation to the initial value of the cost is presented in Table 2 .
Table 2

The cost value decrease, given in [\%]

\begin{tabular}{ccc}
\hline \hline Load case & Topology optimization & Configuration optimization \\
\hline 1 & 19.8 & 30.3 \\
\hline 2 & 25.7 & 29.7 \\
\hline 1 and 2 & 19.8 & 25.4 \\
\hline
\end{tabular}

\section{Conclusions}

The heuristic algorithm of optimization of trusses is presented and tested in this paper. Here, topology optimization is performed using the bars exchange method. It provides a new and effective tool of an optimal design of trusses. The characteristic feature of this approach is the possibility of simultaneous generation of many equivalent optimal topologies, which especially appear for symmetric trusses or trusses containing repeated bar subsystems. Moreover, bar forces for a new topology are calculated using the previous solution, so this method ensures a significant reduction of the computational time.

\section{REFERENCES}

[1] U. Kirsch, "Integration of reduction and expansion processes in layout optimization", Struct. Optim. 11, 13-18 (1996).

[2] W.S. Dorn, R.E. Gomory, and H.J. Greenberg, "Automatic design of optimal structures", J. de Mecanique 3, 25-52 (1964).

[3] U. Kirsch, "Optimal topologies of truss structures", Appl. Mech. Rev. 42, 223-239 (1989).

[4] G.I.N. Rozvany, "Topology optimization in structural mechanics", in CISM Courses and Lectures 374, Springer, Wien, 1967.

[5] W. Achtziger, "On simultaneous optimization of truss geometry and topology", Struct. Multidisc. Optim. 33, 285-304 (2007).

[6] D. Bojczuk and Z. Mróz, "Optimal design of trusses with account for topology variation”, Mech. Struct. \&Mach. 26, 21-40 (1998).

[7] J.J. McKeown, "Growing optimal pin-jointed frames", Struct. Optim. 15, 92-100 (1998).

[8] T. Hagishita and M. Ohsaki, "Topology optimization of trusses by growing ground structure method", Struct. Multidisc. Optim. 37, 377-393 (2009).

[9] D. Bojczuk, Sensitivity Analysis and Optimization of Bar Structures, Publishing House of Kielce University of Technology, Kielce, 1999, (in Polish).

[10] Z. Mróz and D. Bojczuk, "Finite topology variations in optimal design of structures", Struct. Multidisc. Optim. 25, 153-173 (2003).

[11] G. Leugerging and J. Sokolowski, "Topological sensitivity analysis for elliptic problems on graphs", Control and Cybernetics 37 (4), 971-997 (2008).

[12] G.I.N. Rozvany, "On symmetry and non-uniqueness in exact topology optimization", Struct. Multidisc. Optim. 43, 297-317 (2011).

[13] D. Bojczuk, "Sensitivity analysis of elastic bar and beam structures", Eng. Transactions 35, 171-204 (1987), (in Polish).

[14] Z.P. Bazant and L. Cedolin, Stability of Structures, Oxford University Press, Oxford, 1991. 